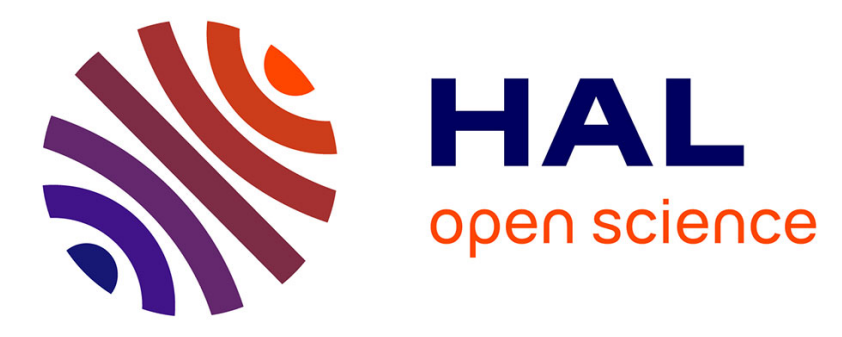

\title{
Lamb-shift and electric field measurements in plasmas
}

Fabrice Doveil, Laurence Chérigier-Kovacic, Peter Ström

\section{To cite this version:}

Fabrice Doveil, Laurence Chérigier-Kovacic, Peter Ström. Lamb-shift and electric field measurements in plasmas. Plasma Physics and Controlled Fusion, 2016, Special issue featuring the invited talks from the 43rd EPS Conference on Plasma Physics, Leuven, 4-8 July 2016, 59 (1), 10.1088/0741$3335 / 59 / 1 / 014020$. hal-01466155

\section{HAL Id: hal-01466155 \\ https://hal-amu.archives-ouvertes.fr/hal-01466155}

Submitted on 16 Feb 2017

HAL is a multi-disciplinary open access archive for the deposit and dissemination of scientific research documents, whether they are published or not. The documents may come from teaching and research institutions in France or abroad, or from public or private research centers.
L'archive ouverte pluridisciplinaire $\mathbf{H A L}$, est destinée au dépôt et à la diffusion de documents scientifiques de niveau recherche, publiés ou non, émanant des établissements d'enseignement et de recherche français ou étrangers, des laboratoires publics ou privés. 
Lamb-shift and electric field measurements in plasmas

This content has been downloaded from IOPscience. Please scroll down to see the full text.

2017 Plasma Phys. Control. Fusion 59014020

(http://iopscience.iop.org/0741-3335/59/1/014020)

View the table of contents for this issue, or go to the journal homepage for more

Download details:

IP Address: 83.156.67.191

This content was downloaded on 21/01/2017 at 09:05

Please note that terms and conditions apply.

You may also be interested in:

Lyman- radiation of a probe atomic beam to explore the electric field in plasma sheaths

Aurélien Lejeune, Laurence Chérigier-Kovacic and Fabrice Doveil

Dust as fine electrostatic probes

A A Samarian and B W James

Experimental investigation of the relation between $\mathrm{H}$ negative ion density and Lyman- emission intensity in a microwave discharge

S Aleiferis, O Tarvainen, $P$ Svarnas et al.

$\underline{X \text {-ray laser spectroscopy of highly charged ions at FLASH }}$

S W Epp, J R Crespo López-Urrutia, M C Simon et al.

Extra physics with an ABS and a Lamb-shift polarimeter

$R$ Engels, $R$ Emmerich, $K$ Grigoryev et al.

Electric field measurements in a hollow cathode

M I de la Rosa, C Pérez, K Grützmacher et al.

Atom based RF electric field sensing

Haoquan Fan, Santosh Kumar, Jonathon Sedlacek et al.

Observation of Stark Spectra of Argon High Rydberg States in Well-Defined Electric Fields by Laser-Induced Fluorescence-Dip Spectroscopy

Kazuki Takizawa, Koichi Sasaki and Kiyoshi Kadota

Coherent beam--foil excitation of $2 \mathrm{~s} 1 / 2$ and $2 \mathrm{p} 1 / 2$ states of hydrogen and antihydrogen atoms

L Labzowsky, V Sharipov, D Solovyev et al. 


\title{
Lamb-shift and electric field measurements in plasmas
}

\author{
F Doveil $^{1}$, L Chérigier-Kovacic ${ }^{1}$ and $\mathbf{P}$ Ström ${ }^{2}$ \\ 1 Aix-Marseille Université, CNRS, PIIM, UMR 7345, case 321, campus Saint-Jérôme, FR-13013 \\ Marseille, France \\ ${ }^{2}$ Department of Fusion Plasma Physics, Royal Institute of Technology (KTH), SE-100 44 Stockholm, Sweden \\ E-mail: fabrice.doveil@univ-amu.fr and laurence.kovacic@univ-amu.fr
}

Received 30 June 2016, revised 29 August 2016

Accepted for publication 9 September 2016

Published 18 October 2016

\begin{abstract}
The electric field is a quantity of particular relevance in plasma physics. Indeed, its fluctuations are responsible for different macroscopic phenomena such as anomalous transport in fusion plasmas. Answering a long-standing challenge, we offer a new method to locally and non-intrusively measure weak electric fields and their fluctuations in plasmas, by means of a beam of hydrogen ions or atoms. We present measurements of the electric field in vacuum and in a plasma where Debye shielding is measured. For the first time, we have used the Lamb-shift resonance to measure oscillating electric fields around $1 \mathrm{GHz}$ and observed the strong enhancement of the Lyman- $\alpha$ signal. The measurement is both direct and non-intrusive. This method provides sensitivity $\left(\mathrm{mV} \mathrm{cm}^{-1}\right)$ and temporal resolution (ns) that are three orders higher compared to current diagnostics. It thus allows measuring fluctuations of the electric field at scales not previously reached experimentally.
\end{abstract}

Keywords: basic plasma physics, Debye shielding, plasma sheath, Lamb shift, Stark mixing

(Some figures may appear in colour only in the online journal)

\section{Introduction}

Recently, a new non-intrusive diagnostic was proposed to locally measure static and fluctuating electric fields in a plasma [6]. This addresses a longstanding challenge: to precisely measure electric fields in plasmas, where they play a key role. For example, static electric fields are at the root of the formation of sheaths and their prominent role in plasma surface interactions is well known. Direct measurements of electric field would also be interesting for plasma thrusters [22]. Fluctuating electric fields are the main cause in plasma turbulence leading to nonlinear wave-particle interactions and so called anomalous transport. Many experimental methods have been developed. Whenever possible, electrostatic probes are widely used but always cause a significant perturbation of the surrounding plasma [18]. In specific discharges dust particles levitating in a plasma can also be used but the electric field measurement relies heavily on good knowledge of the dust charging process [19]. In the plasma core of tokamaks, injection of a heavy singly charged ion beam creates doubly charged ions the measurement of whose energy gives information on the plasma potential at the ionization point, and thus indirect electric field measurement [21]. The effect of the field on the motion of charged particles can also be recorded using laser induced fluorescence techniques and the electric potential in the plasma is indirectly derived from the shift of the measured velocity distribution function [2]. Other optical non intrusive methods have been developed but, except laser Stark spectroscopy working for high amplitude electric fields, these methods cannot be considered as direct and need elaborate and rather expensive tools. This noncomprehensive list urges the need for a reliable diagnostic by which the local electric field amplitude can be deduced directly from the measured physical quantity.

The measurement of a plasma sheath between two conducting plates in an argon discharge and the measurement of a fluctuating field and comparison with the theoretical prediction in our experimental conditions are two complementary results. They exhibit that this innovative diagnostic provides a very useful and efficient way to measure low amplitude electric fields in vacuum and plasmas. 


\section{Principle of the diagnostic}

Our non-intrusive diagnostic called EFILE (electric field induced Lyman- $\alpha$ emission) is based on the Lyman- $\alpha$ emission of a probing hydrogen atomic beam. Since the radiative corrections were introduced in quantum physics through QED theory established in the early 1960 s through to 1975 , the $2 s_{1 / 2}$ and $2 p_{1 / 2}$ levels in the atomic fine structure are said to be neardegenerate for hydrogen atoms. The degeneracy is removed by small quantum electrodynamic effects, known as radiative corrections, due to interactions between the electron and electromagnetic fluctuations of vacuum. They are responsible for energy shifts of levels called Lamb-shifts. It was experimentally shown by Lamb [10] that, the $2 s_{1 / 2}$ state does not have exactly the same energy as the $2 p_{1 / 2}$ state. It lies higher by a small amount $h \nu_{0}$ where $h$ is the Planck constant and $\nu_{0}=1057 \mathrm{MHz}$ is the resonance frequency corresponding to the Lamb-shift for H(2s). This effect has been studied extensively since then, both experimentally to precisely measure the shift $[10]$ and theoretically to understand its origins $[9,11]$.

The $2 s_{1 / 2}$ level is metastable, the transition to the ground state $1 s_{1 / 2}$ being forbidden. The lifetime of $2 s_{1 / 2}$ is about $0.14 \mathrm{~s}$, which is very long compared to $1.6 \times 10^{-9} \mathrm{~s}$, the lifetime of the $2 p_{1 / 2}$ state [11]. In the presence of an externally applied electric field, quenching of the metastable $2 s$ state of hydrogen and hydrogen-like atoms leads to the production of Lyman- $\alpha$ radiation with number of photons per second $I_{\alpha}=\Omega V n_{2 s} \gamma_{2 s}^{*}$ where $\Omega$ is a geometric factor, $V$ is the interacting volume, $n_{2 s}$ is the $2 s$ metastable hydrogen atom density and $\gamma_{2 s}^{*}$ is the quenched $2 s$ transition rate related to the reduced lifetime, resulting from so-called Stark mixing between the $2 s_{1 / 2}$ and $2 p_{1 / 2}$ levels. A weak electric field introduces a perturbation to the full energy of an electron in a given state. Here, we consider the states $2 s_{1 / 2}$ and $2 p_{1 / 2}$. The perturbation approach is valid as long as the Stark shift that the electric field gives rise to is small in comparison to the energy difference between the studied levels. At $475 \mathrm{~V} \mathrm{~cm}^{-1}$ constant field, the Stark shift is of the same order of magnitude as the Lamb shift, and the perturbation approach is therefore valid only for electric field strengths significantly below that magnitude. Bethe and Salpeter [5] have derived equations for the populations of two levels having the same value of quantum numbers $n$ and $j$ in low electric fields. Crandall and Jaecks [7] can be followed in order to calculate the decay rate $\gamma_{2 s}^{*}$ of the $2 s_{1 / 2}$ level to the ground level (details of calculation can be found in more recent works like $[3,4,20])$

$$
\gamma_{2 s}^{*}(\omega)=\frac{9 e^{2} a_{0}^{2} E_{0}^{2}}{\hbar^{2}} \frac{\gamma_{2 p}}{\left(\omega-\omega_{0}\right)^{2}+\gamma_{2 p}^{2} / 4},
$$

where $\omega=2 \pi \nu$ and $\omega_{0}=2 \pi \nu_{0}$. In the limit of a constant field, we find Lamb's result [11] with a quadratic dependence of the measured Lyman- $\alpha$ radiation versus electric field amplitude. At the resonance corresponding to an oscillating electric field with frequency $\nu=\nu_{0}$, the above calculation gives an amplification factor of more than two orders of magnitude, which is independent of the value of the field. All these predictions were confirmed by our previous measurements for an electric

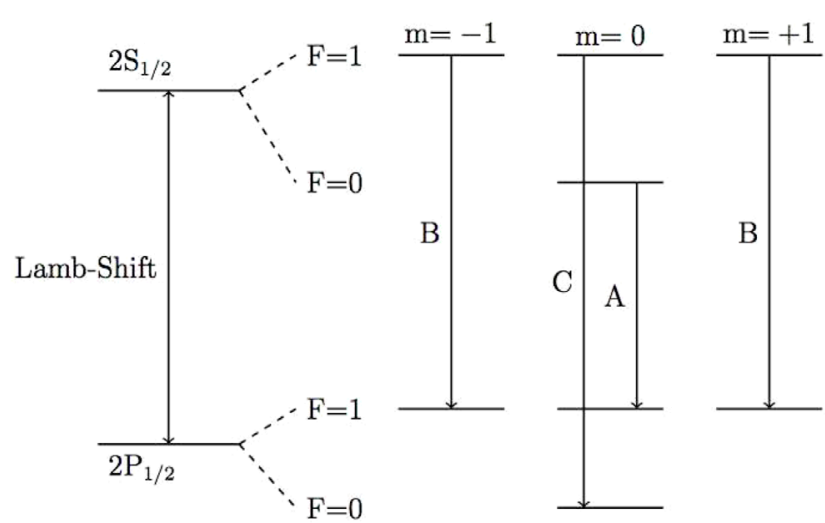

Figure 1. Hyperfine states and transitions: $A=909.67 \mathrm{MHz}$, $B=1087.4 \mathrm{MHz}, C=1146.6 \mathrm{MHz}$.

field created between two biased parallel plates in vacuum $[6,8,12-14,17]$.

One can also take into account hyperfine structure effects. With more energy levels, we then have to consider three possible transitions between hyperfine states induced by a perturbation in the form of a sinusoidal or constant electric field, as shown in figure 1. For the final expression of the transition rate from $2 s_{1 / 2}$ to the ground state, all hyperfine state pairs indicated in figure 1 are considered. Summing the rates due to the Stark mixing of every individual pair and considering all resonance frequencies to be the same should reproduce the rate given by equation (1). It is assumed that all pairs contribute equally to the total rate. This means that every pair gives one fourth of the expression of equation (1) with $\omega_{0}$ replaced by the appropriate resonant pulsation. Figure 2 shows the resulting theoretical frequency dependence of the total transition rate and the individual pairs at an arbitrary value $E_{0}=3 \mathrm{~V} \mathrm{~cm}^{-1}$.

The transition rate is expected to peak around the resonant frequencies. Note however that the lines do have a natural width, and as a result only the hyperfine separation of the $2 s$ level is seen in the plot. The separation of $2 p$ is smaller than the natural linewidth and it should not be possible to see it without using a refined method [15].

The practical use of the above-mentioned principle showing that Lyman- $\alpha$ emission intensity of a test hydrogen beam is related to the square amplitude of a perturbing constant or sinusoidal electric field could be tested in the experimental set-up briefly presented below.

\section{Description of the diagnostic}

The experimental set-up has been described previously $[6,8,13,17]$. Figure 3 shows a picture of the experimental set-up and the main parts of the apparatus with the beam generation part, the measurement region and the detector. The atomic beam originates in a proton beam extracted from a multipolar hydrogen plasma source, labelled $A$ on the photo of figure 3. It is created either by collisions with $\mathrm{H}_{2}$ residual gas along the beam path, or by resonant charge exchange in a cell containing cesium vapor [1], labelled $B$ on the photo of figure 3 . We work with a $500 \mathrm{eV}$ ion beam, which corresponds to the maximum cross section of this charge exchange process [16]. 


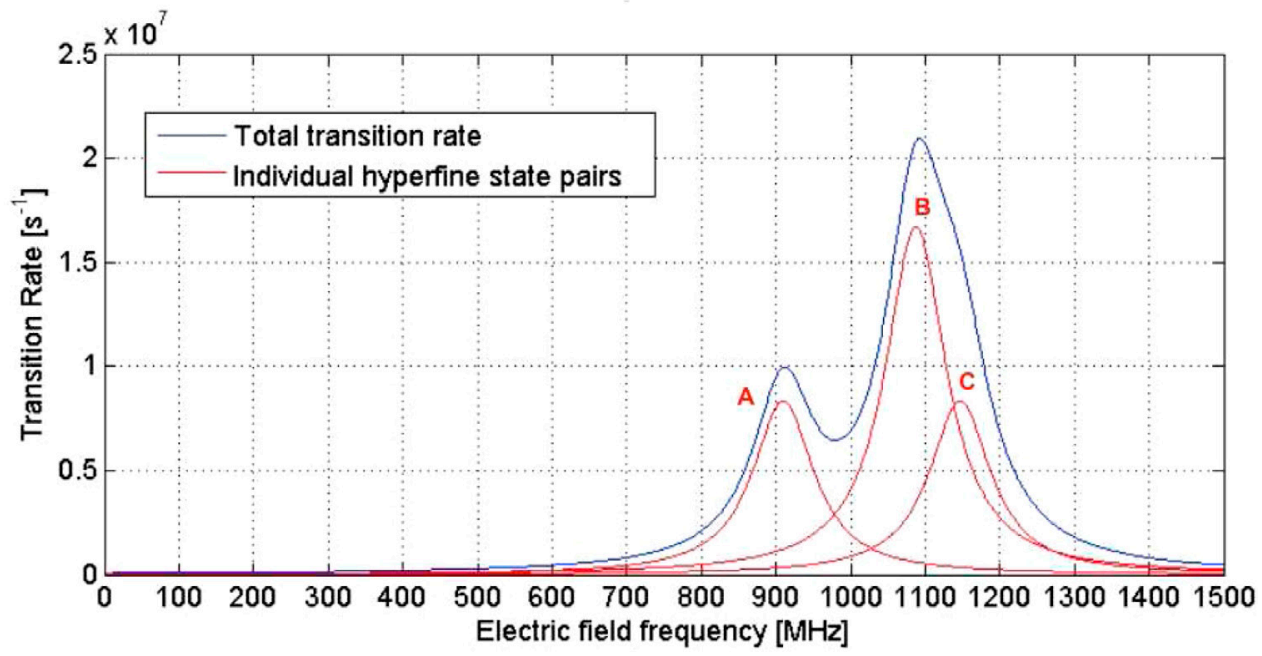

Figure 2. Total transition rate.
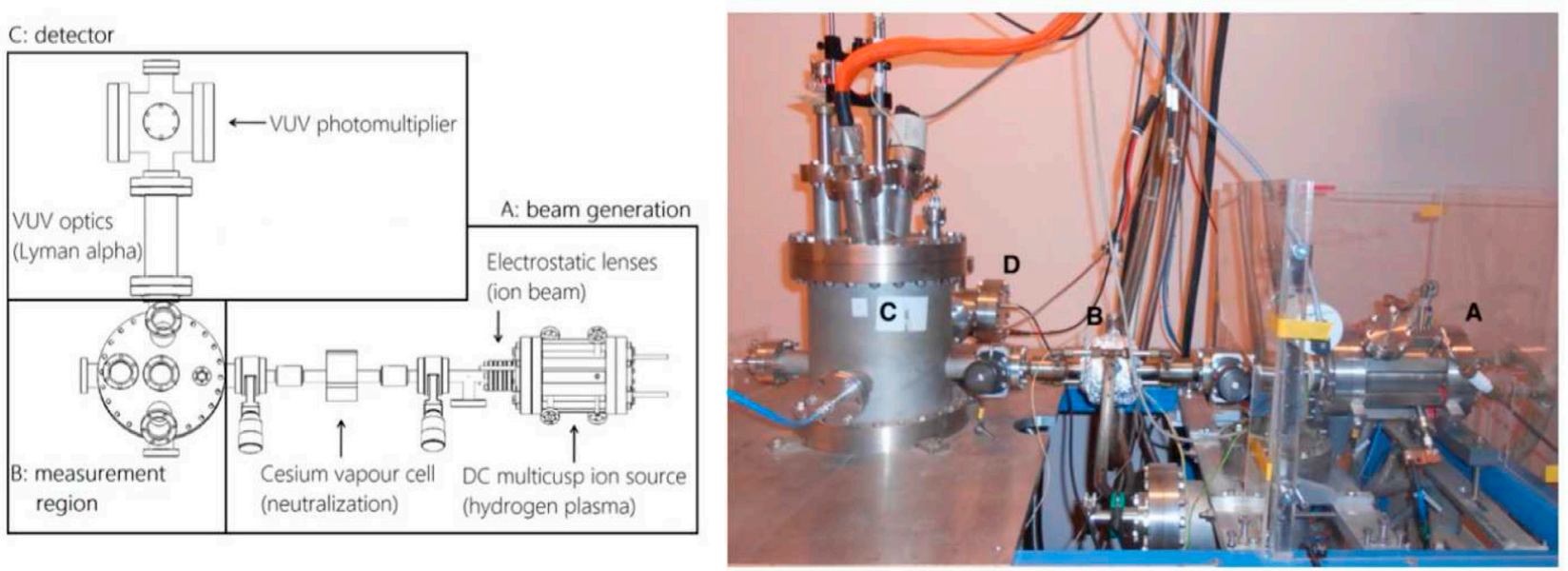

Figure 3. Scheme and photo of the experimental setup.

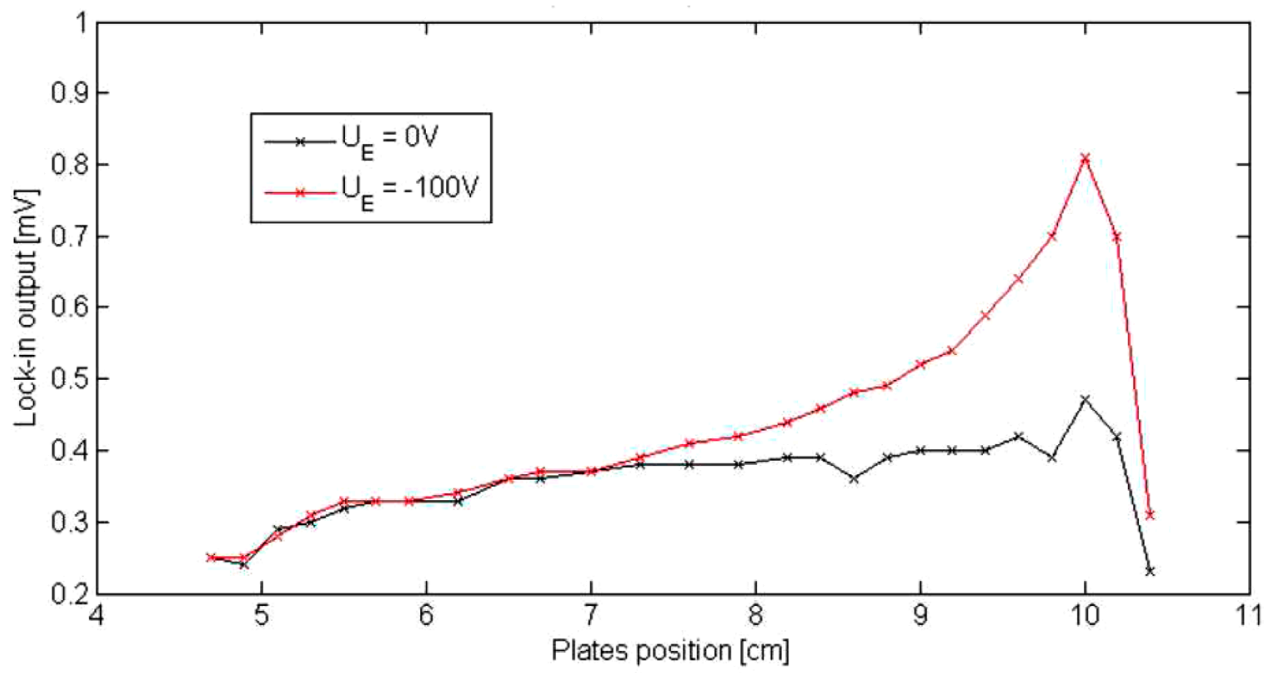

Figure 4. Electric field profile in a plasma: raw data $I_{d}=0.95 \pm 0.01 \mathrm{~A}, U_{d}=80 \mathrm{~V}, p=(1.8 \pm 0.2) \cdot 10^{-5} \mathrm{mbar}, I_{d}^{\prime}=0.26 \pm 0.05 \mathrm{~A}$, $U_{d}^{\prime}=50 \mathrm{~V}, p^{\prime}=(5.1 \pm 0.2) \cdot 10^{-4}$ mbar.

The beam is directed into a measurement chamber, labelled $C$ on the photo of figure 3. The Lyman- $\alpha$ light $(\lambda=121.6 \mathrm{~nm})$ is collected in a direction perpendicular to the beam through a lithium fluoride $(\mathrm{LiF})$ lens and is detected by a VUVphotomultiplier operating in vacuum, labelled $D$ on the photo of figure 3. Spatial resolution is determined solely by the size 


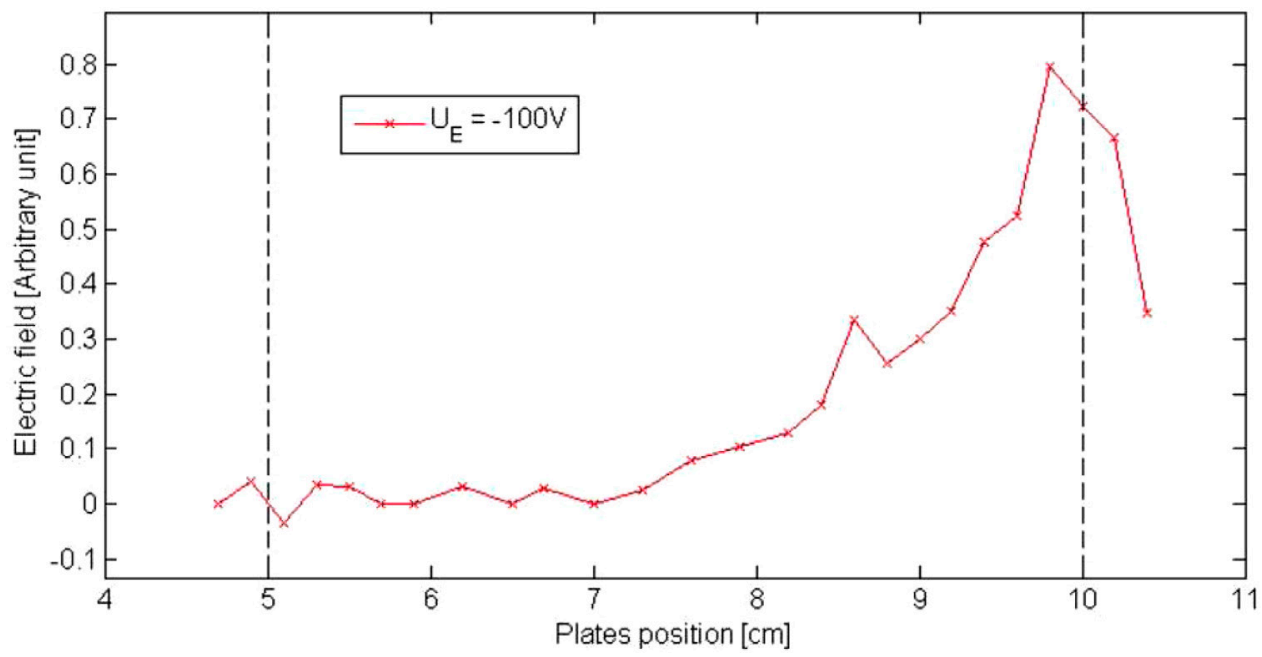

Figure 5. Electric field profile in a plasma.

of the photocathode and the lens magnification. In order to improve the signal-to-noise ratio, a lock-in amplifier is used with either a modulated ion beam extraction at a very low frequency (typically $1 \mathrm{~Hz}$ ), or with a modulated electric field at a low frequency (typically $1 \mathrm{kHz}$ ). The measured signal corresponds to the integral of the emission over the Lyman- $\alpha$ line. When the external electric field is modulated, the continuous background of spontaneous emission from the beam is not recorded-only the part of the signal induced by the electric field, synchronized with the field modulation, is observed. The hydrogen pressure in the measurement chamber is usually about one tenth of the pressure in the source. This allows us to make measurements even with a pure ion beam through collisions between ions and the residual gas [6]. This pressure can also be varied with an independent argon gas introduction.

Then, a low density DC argon plasma can be produced at low pressure (typically a few $10^{-5}$ mbar) through thermionic emission of a hot tungsten filament biased negatively (typically $-80 \mathrm{~V}$ ) with respect to the grounded walls of the target chamber. In this target chamber, two horizontal parallel plates surrounding the beam and separated by $5 \mathrm{~cm}$ can be externally biased to create a static (or oscillating) electric field. One plate is grounded while the other can be biased, either at a constant voltage or at an RF fluctuating voltage. The profile of the electric potential or related field is obtained by moving the biased plates vertically around the plasma-beam interacting volume.

\section{Static electric field in a plasma sheath}

We first consider the case of a static electric field between the two parallel plates. For the lock-in detection, the hydrogen beam is pulsed at a frequency of $1 \mathrm{~Hz}$. We have recorded the electric field profile between the plates by moving both of them vertically with respect to the fixed beam and measuring the lock-in output of the VUV-photomultiplier signal at up to 28 points. This has been done for several static voltage settings from $-100 \mathrm{~V}$ to $0 \mathrm{~V}$ in a set of different plasmas. An illustrative example of raw data is presented in figure 4. Pressure, discharge current and voltage $I_{d}, U_{d}$ in the source and $I_{d}^{\prime}, U_{d}^{\prime}$ in

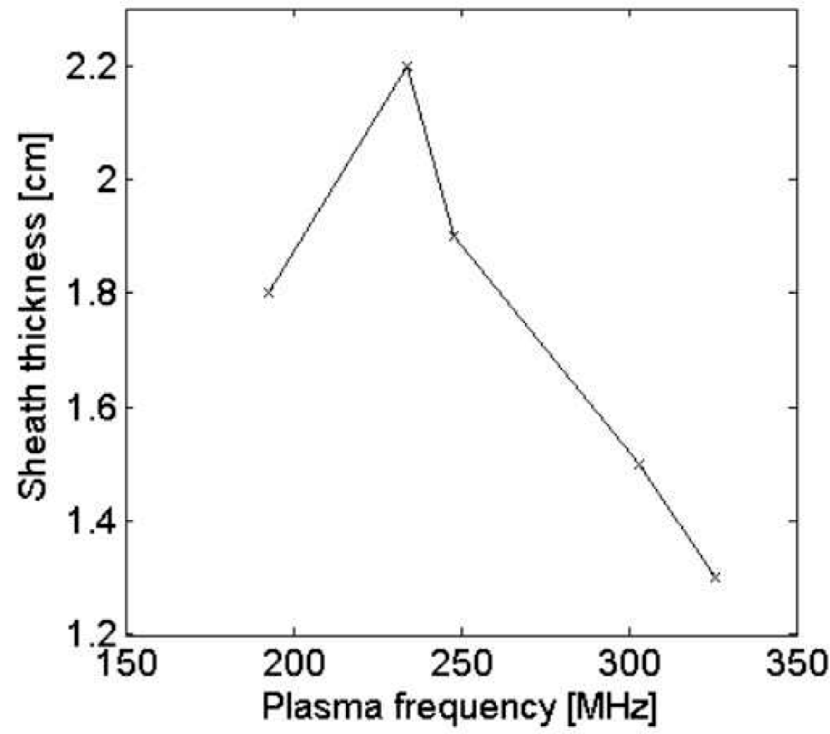

Figure 6. Sheath thickness versus plasma frequency $I_{d}=0.95 \pm 0.01 \mathrm{~A}, U_{d}=80 \mathrm{~V}, p=(1.8 \pm 0.2) \cdot 10^{-5} \mathrm{mbar}$, $I_{d}^{\prime}=0.13-0.65 \pm 0.05 \mathrm{~A}, U_{d}^{\prime}=100 \mathrm{~V}, p^{\prime}=(1.1 \pm 0.1) \cdot 10^{-3} \mathrm{mbar}$.

the measurement chamber are given in the figure caption. The points around $5 \mathrm{~cm}$ correspond to the upper (grounded) plate being lowered into and past the beam, and the points at $10 \mathrm{~cm}$ give the same for the lower (biased) plate.

In a previous paper [6], we have demonstrated that it is possible to use collected data to reconstruct sought profiles in the static case. The electric field profiles in vacuum thus produced agree with those obtained via numerical calculation, apart from explainable differences that are specific to our setup. This gives a reliable calibration for electric field measurements through the recorded Lyman- $\alpha$ intensity. Static profiles in a plasma, such as those shown in figure 4, are measured for a variety of different values of the plasma parameters that we can vary. They display the expected behaviour with a strong field in the plasma sheath.

This is better shown in figure 5, where we plot the compensated profile obtained from the relative difference $(S($ Voltage $)-S(0 \mathrm{~V})) / S(0 \mathrm{~V})$ of the raw curves in figure 4. 


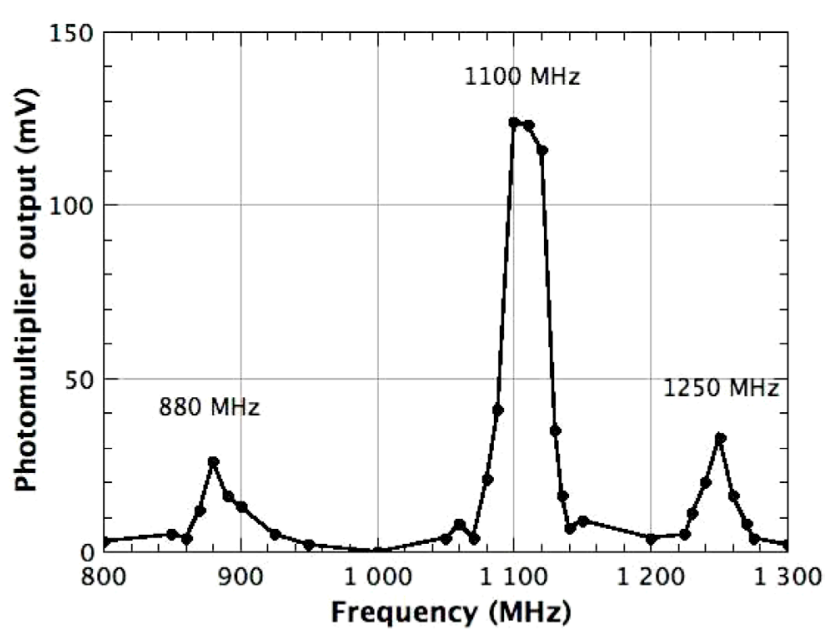

Figure 7. Photomultiplier signal as a function of the electric field frequency.

Outside the sheath (or to be specific outside the presheath), the data with and without voltage at the lower plate coincide almost exactly, verifying that the electric field is close to zero there. Note that the electric field seems to drop at $10 \mathrm{~cm}$, as if the sheath were separated from the biased plate. This is unphysical and due to geometrical saturation for strong signals as described in [6].

For a variety of target plasma conditions (discharge parameters in source and measurement chambers given in the figure caption), we were able to record the plasma frequency and its harmonics from the RF signal of the entrance grid of the energy analyzer (previously used to analyze the hydrogen ion beam production) collected on a spectrum analyzer. The sheath width, as measured from figure 5, is presented in figure 6 as a function of plasma frequency. This measurement is consistent with the well-known result of a sheath several Debye lengths thick, strengthening the statement that our electric field measurement method is applicable for static fields in a plasma. The drop in sheath width for the point close to $f_{\text {plasma }}=200 \mathrm{MHz}$ is explained by a strong decrease in electron temperature as this point is very close to the limit at which the discharge is lost.

\section{RF electric field}

An RF field is produced in the measurement chamber using the biased plate as an emitting antenna. For the lock-in detection, the beam is now continuous and the RF field is modulated in amplitude at a low frequency around $1 \mathrm{kHz}$, thus suppressing any background Lyman- $\alpha$ emission other than due to the RF field. In a previous paper, we verified the enhancement predicted by equation (1) when the frequency of the applied electric field is resonant with the Lamb shift frequency and thus proved the great capacity of our method to measure weak electric fields. We keep the RF electric field amplitude constant and vary its frequency around the Lamb shift frequency while recording the lock-in output (proportional to the square of the field modulus), expecting to retrieve the results of figure 2. The result is displayed in figure 7 . It is remarkable that the observed spectrum exhibits three peaks: two of these $(880 \mathrm{MHz}$ and $1100 \mathrm{MHz}$ ) can be related to the hyperfine structure of $\mathrm{H}(2 \mathrm{~s})$ hydrogen atoms as reported in section 2. The third, at $1250 \mathrm{MHz}$, corresponds to a transition between $4 s_{1 / 2}$ (which has, like $2 s_{1 / 2}$ a quite long lifetime of 23 $\mu \mathrm{s})$ and $4 p_{3 / 2}$. However we also notice that the measured peaks are much narrower than predicted in figure 2 .

We have reproduced such frequency spectra for different measurement chamber geometries: presence or not of an energy analyzer and its supporting rod, presence or not of an extra antenna and its supporting rod, presence of a filament. In each case, we have obtained similar spectra but with slightly different positions and amplitudes of the peaks. We have also used an extra antenna and measured the signal received on this antenna. We have observed many peaks in the frequency range of interest, which are associated to the resonance modes of the target chamber-actually being a cavity with a complex geometry. These peaks having a similar width to the lines in figure 7 , our conclusion is that this constitutes the main explanation for this result.

\section{Conclusion}

This paper provides further insight into the capability of our new non-intrusive diagnostic EFILE to accurately measure local static or fluctuating electric fields. It must be stressed that the diagnostic is based on thoroughly established underlying science and uses a fairly current technology for the formation of the $\mathrm{H}(2 \mathrm{~s})$ beam.

Beside providing an efficient way to measure low amplitude electric fields - as low as $1 \mathrm{~V} \mathrm{~cm}^{-1}$ — the diagnostic can acquire a still better spatial resolution by reducing the waist of the beam. Signal should also be easily improved by removing the remaining $\mathrm{H}_{2}^{+}, \mathrm{H}_{3}^{+}$, and $\mathrm{H}^{-}$ions of the beam with extra magnetic field.

In the near future, the definite calibration of RF field amplitude will be completed. The influence of an extra magnetic field will be studied and the possibility of measuring local magnetic field through unfolding of the motional $v \times B$ field will be explored. Comparison with existing LIF measurements in a plasma will be performed. The possibility of completing the photomultiplier measurements with emission spectroscopy measurements will also be assessed.

\section{Acknowledgments}

The authors are grateful to J-B Faure, J-P Busso for technical assistance. They acknowledge the help of G Bachet and G Prasad for the conception and construction of the experimental set-up. P.S. benefited from an Erasmus exchange grant via the European Lifelong Learning Programme 2007-2013.

\section{References}

[1] Bacal M et al 1974 Nucl. Inst. and Methods 114407 Bacal M et al 1974 Rev. Sci. Instrum. 45769

[2] Bachet G, Chérigier L, Carrère M and Doveil F 1993 Phys. Fluids B 53097 
[3] Benage J F Jr 1986 Plasma effects on the metastable $\mathrm{H}(2 s)$ atoms PhD Thesis University of Colorado

[4] Benage J F Jr, Stevens R R Jr and Stern R A 1989 Nucl. Instrum. Methods A 27413

[5] Bethe H A and Salpeter E E 1957 Quantum Mechanics of One and Two-Electron Atoms (Berlin: Springer)

[6] Chérigier-Kovacic L, Ström P, Lejeune A and Doveil F 2015 Rev. Sci. Instrum. 86063504

[7] Crandall D H and Jaecks D H 1971 Phys. Rev. A 42271

[8] Doveil F, Lejeune A and Chérigier-Kovacic L 2013 Phys. Plasmas 20055701

[9] Lamb W E Jr 1951 Rep. Prog. Phys. 1419

[10] Lamb W E Jr and Retherford R C 1947 Phys. Rev. 72241

[11] Lamb W E Jr and Retherford R C 1950 Phys. Rev. 79549

[12] Lejeune A 2010 Mise au point d'un diagnostic optique non intrusif pour la mesure des micro-fluctuations locales des champs électriques dans les plasmas Thèse de Doctorat Université de Provence

[13] Lejeune A, Chérigier-Kovacic L and Doveil F 2011 Appl. Phys. Lett. 99181502
[14] Lejeune A, Chérigier-Kovacic L and Doveil F 2008 Europhysics Conf. Abstracts 35th Conf. on Contr. Fusion and Plasma Physics vol 32D (Hersonissos: European Physical Society) P1.186

[15] Lundeen S R, Jessop P E and Pipkin F M 1975 Phys. Rev. Lett. 34377

[16] Pradel P, Roussel F, Schlachter A S, Spiess G and Valance A 1974 Phys. Rev. A 10797

[17] Prasad G, Bachet G, Doveil F, Chérigier L and Stern R A 1998 Europhysics Conf. Abstracts of 1998 ICPP and 25th Conf. on Contr. Fusion and Plasma Physics vol 22C (Praha: European Physical Society) p 110

[18] Ratinskaia S V, Demidov V I and Rypdal K 2002 Phys. Plasmas 94135

[19] Samarian A A and James B W 2005 Plasma Phys. Control. Fusion 47 B629

[20] Ström P 2013 MS Thesis Université d'Aix Marseille (http:// urn.kb.se/resolve?urn=urn:nbn:se:uu:diva-206123)

[21] Yang X Z et al 1991 Phys. Fluids B 33448

[22] Dudeck M, Doveil F, Arcis N and Zurbach S 2012 IOP Conf. Ser.: Mater. Sci. Eng. 29012010 